\title{
Fault Detection and Diagnosis of the Valve Actuators in HVAC Systems, Using Frequency Analysis
}

\author{
N. TUDOROIU* and M. ZAHEERUDDIN** \\ * Department of Electrical and Computer Engineering, Concordia University, Montreal, CA \\ E-mail: tnicolae@excite.com \\ **Department of Building, Civil and Environmental Engineering, Concordia University, Montreal, CA \\ E-mail: zaheer@cbs-engr.concordia.ca
}

\begin{abstract}
One of the main objectives of our research is to build several ARMA linear models and nonlinear neuro models for DAT systems such as developed in [3], [4]. Based on these models we have been interested to build some feedback control strategies for these systems [3]-[5]. Due to the highly complexity of the HVAC systems the requirements for reliability, availability, and security grow significantly and consequently investigations in this field become necessary. The objective of our paper is to develop some strategies for the fault detection and diagnosis (FDD) of the HVAC systems based on frequency and spectral analysis of the system response. The main interest is focused on dealing with the unanticipated valves actuators failures in the most general formulation based on the frequency analysis of the system response. In our study we consider that the increasing of the backlash opening in the valve actuators could create serious problems by degrading drastically the HVAC system performance. Based on the spectrum analysis we are capable to gather the most significant patterns that characterize well each fault mode detected, and finally a systematic procedure and technique for proper fault accommodation under the unanticipated failures will be developed. As tools we use MATLAB with SIMULINK toolboxes from control systems, system identification and signal processing.
\end{abstract}

\section{INTRODUCTION}

Modern HVAC systems failures caused by unexpected interference, or aging of system components will possibly result in changes or changing of system dynamics.

Due to the highly complexity of the HVAC systems the requirements for reliability, availability, and security grow significantly. To meet these requirements there is a need for robust fault detection and diagnosis tools capable to detect and isolate any sensor, actuator or system components subjected to failure as soon and as accurately as possible.

To regulate parameters such as temperature, humidity, pressure and flow rates in HVAC systems in commercial buildings several conventional SISO PI/PID, self-tuning and adaptive feedback control strategies are used. The closedloop control design for improving the performance of heating, ventilating, and air-conditioning (HVAC) systems is a very difficult task because the HVAC systems exhibit inherent nonlinear behavior, have a multivariable structure, and are acted upon by multiple disturbances [2] -
[5]. In this paper we consider the cooling coil as a SISO object, controlled by an PI strategy and we will explore how to build a corresponding FDD strategy of the valve actuator failures based on the frequency response analysis of the discharge air temperature (DAT) system which is an important part of all HVAC systems. In the DAT system air entering the heating coil is heated by using hot water. The hot water flow rate is modulated via a valve and actuator in order to maintain the discharge air temperature at the desired set point

The model of the DAT system is highly nonlinear, and the PI controller parameters where tuned for specific values that assure good tracking and dynamic performance for healthy DAT system in closed-loop.

Assuming different scenarios for the evolution of the valve backlash opening we will be capable to detect, diagnose and isolate the fault valve actuator and so remedial action may be undertaken in the shortest time possible.

- Compared to the other FDD strategies developed in HVAC literature, the strategy developed in this paper is more simple and much easier to apply with the possibility of extension to other possible applications from different fields.

\section{DAT SYSTEM-ORIENTED STRUCTURE}

The DAT system could be conceptualized either as a multi-input multi-output (MIMO) or as a single-input single-output (SISO) functional block. In this paper we consider only a simplified SISO Discharge Air Temperature (DAT) model such as the one represented in Figure 1. In the new approach this model is nonlinear and its dynamics is described in [3], [5].

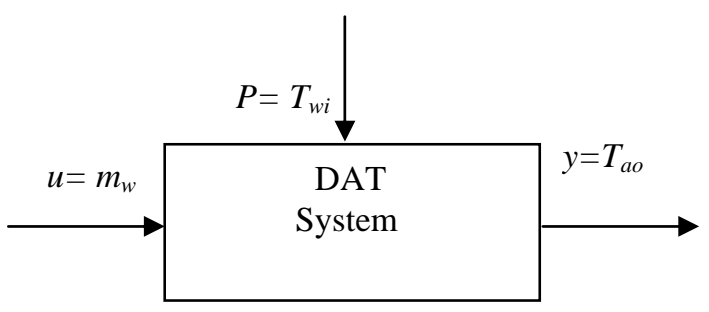

Fig. 1 SISO DAT System -Oriented Structure 
The input of the simplified model (SISO) is hot water flow rate, $m_{w}$ [gpm], $u(k)$, and the output is heating coil output temperature, $T_{a o}\left[{ }^{\circ} \mathrm{C}\right], y(k)$. Hot water supply temperature $T_{w i}\left[{ }^{\circ} \mathrm{C}\right]$ acts as disturbance on the DAT system. The heating coil output temperature is more directly related to the overall DAT system performance, namely tightly regulating it should eliminate much of the variance seen in these systems.

\section{A. The DAT System Representation}

The DAT system is represented in discrete domain by the block scheme from Figure 1. The discrete transfer function of the plant is given by

$$
H_{p}(z)=\frac{0.12}{z^{7}(z-0.95)}
$$

and the PI controller transfer function has the following representation form

$$
H c(z)=\frac{0.04(z-0.01)}{z-1}
$$

The dynamics of the valve actuator is integrated in the plant dynamics, and will be represented by a nonlinear block with backlash characteristics having a variable $\propto$ width.

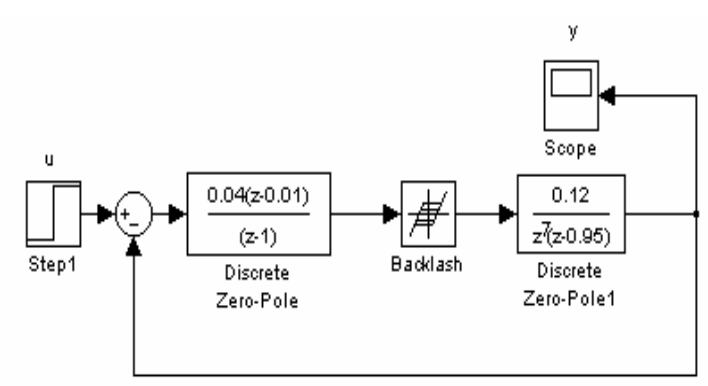

Fig.2 The block scheme of the DAT system

\section{$B$. The step response of the healthy and fault DAT system}

To capture the behavior of the system for different values of the backlash opening $\propto$ we will represent its step responses. The step response of the healthy DAT system ( $\propto$ $=0$ ) is represented in Figure 3, and the step responses for the other different values of $\propto$, such as $\propto=2$, 4, and 6, are represented in Figures 4-6. These figures reveal a progressive degradation of the system performance with the increasing of the backlash width $\propto$. Without to alter the generalization of the fault detection, diagnosis and isolation problem we consider that the last three cases represent real faults in the valve actuator. However the number of the faults could be enlarged, but we are only interested to see how our detection methodology it is working.

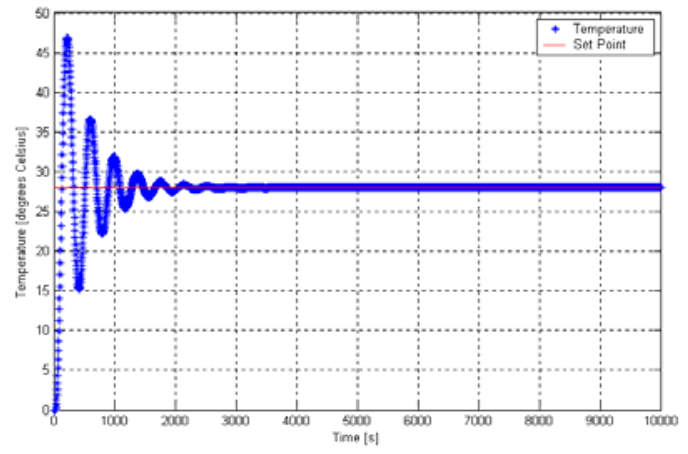

Fig. 2 The evolution of the Temperature for healthy system $(\propto=0)$

Also it is very important to estimate the time necessary to replace the valve before its complete failure.

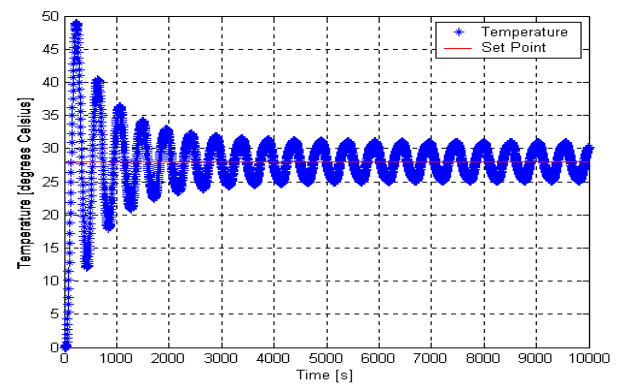

Fig. 3 The evolution of the Temperature for fault system $(\propto=2)$

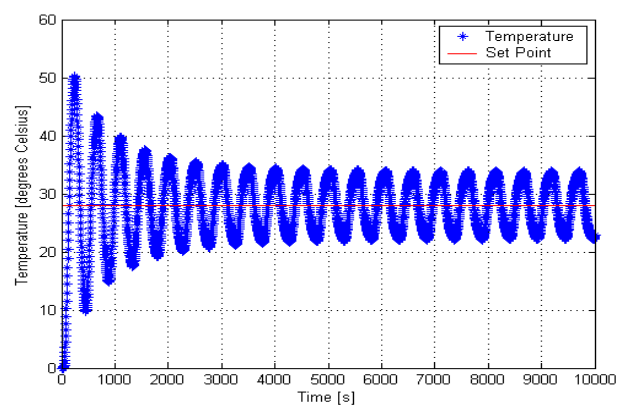

Fig. 4 The evolution of the Temperature for fault system $(\propto=4)$

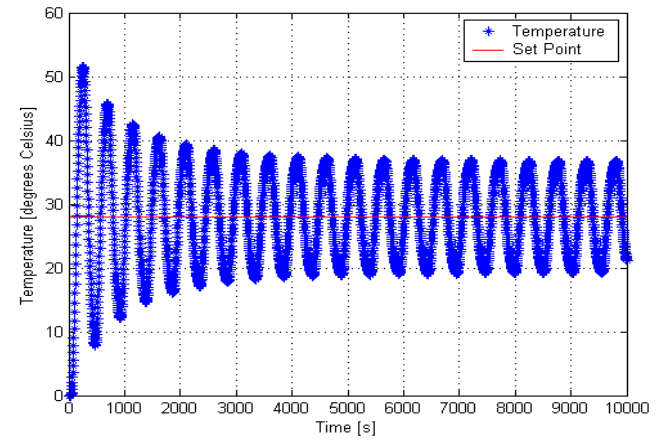

Fig. 5 The evolution of the Temperature for fault system $(\propto=6)$ 


\section{DAT FDD frequency analysis}

The step responses represented in figures 4-6 for different values $\propto$ of the backlash opening reveal the presence of the oscillations that increase in amplitude when $\propto$ increases. The common cause of these oscillations is the presence of the backlash non-linearity which set up a limit cycle in a control loop. The oscillations caused by the faulty valve are liable to act as a disturbance elsewhere in the DAT system. Therefore it is useful to characterize non-linearity in the time trends of the process measurements.

Due to the valve non-linearity the temperature is not steady in such a system and it cycles in periodic patterns.

Sometimes it is more easy to identify similar patterns in the frequency spectrum of the system response compared to the system response in time domain representation.

The spectrum of the system responses for different values $\propto$ of the backlash opening are represented in the figures 7-10.
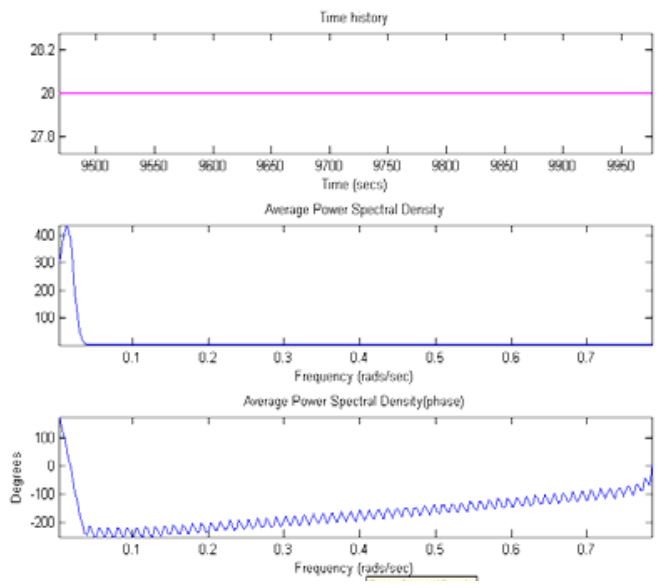

Fig. 6 The time trend and the Power Spectral Density for healthy system
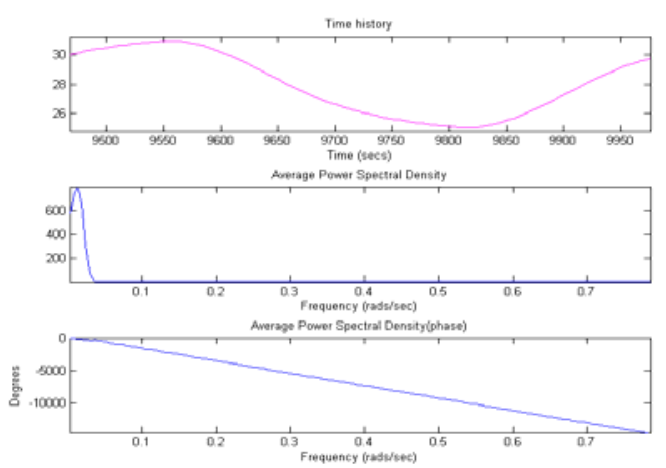

Fig. 7 The time trend and the Power Spectral Density for fault system $(\propto=2)$
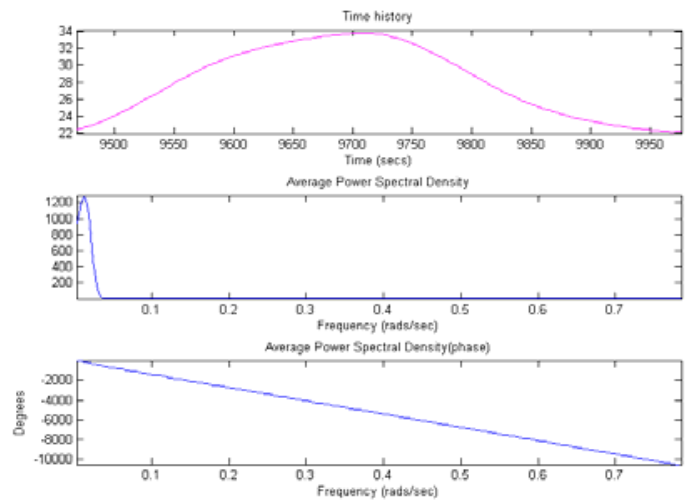

Fig.9 The time trend and the Power Spectral Density for fault system $(\propto=4)$

\section{DETECTION AND DIAGNOSIS OF THE FAULT VALVE ACTUATOR}

In a small study of the HVAC systems the task of finding all measurements influenced by a fault valve may be done by visual inspection.

For an automated approach the spectra of the measurements may be compared to one another using principal components analysis (PCA), well described in [1].

The non-linearity of the valve characteristics may be inferred by the presence of a periodic limit cycles, that are in general non-sinusoidal and consequently have harmonics at multiples of the fundamental frequency.

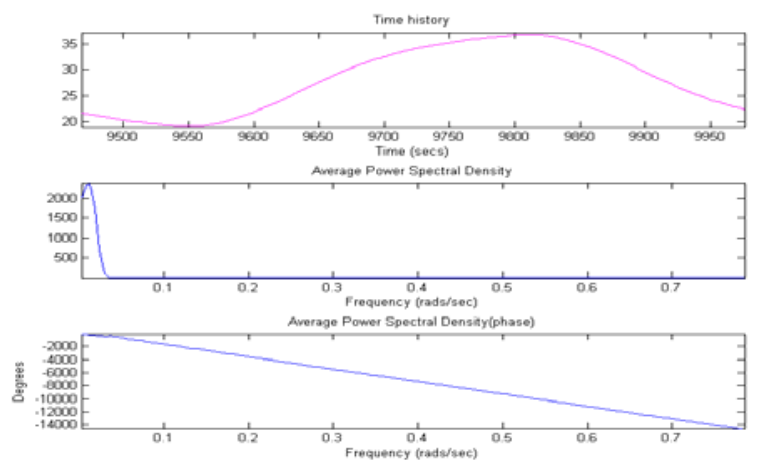

Fig. 8 The time trend and the Power Spectral Density for fault system $(\propto=6)$

If the multi-loop HVAC system oscillations are due to the limit cycle a candidate for the root cause is the measurement with the maximum non-linearity [1].

The reason is that the dynamic behavior of physical processes gives low-pass filtering and therefore removes non-linearity from the time series. As a result, one would expect that the harmonics of a limit cycle to become smaller further from the root cause and the time trends to become more sinusoidal. 
Similar to [1] we can quantify this aspect by calculating the multiplier factor, $D$

$$
D=\sqrt{\frac{P_{a v g, \alpha}}{P_{a v g, 0}}}
$$

where $P_{a v g, 0}$ is the total averaging power spectral density of the DAT system for the healthy valve actuator, $\propto=0$, and $P_{a v g, \alpha}$ is the averaging power spectral density in the fundamental frequency and harmonics of the DAT system for the fault actuator valve.

Calculation of $\mathrm{D}$ requires an inspection of the spectrum represented in the figures (7)-(10) to determine a suitable frequency where the peak value of the averaging power spectral density occurs. The multiplier factor $D$ could be determined only when the oscillation is defined well and the graphs (7)-(10) have power spectral density peaks.

The measurement having the highest multiplier factor has more power in the harmonics and fundamental and so it will be a candidate for root case.

The curves from figures (7)-(10) reveal that if the multiplier factor has the highest value then the value of the $\propto$ parameter is the highest too. For our case the values of the distortion factor are given in the Table 1.

\begin{tabular}{|c|c|}
\hline $\begin{array}{c}\text { Value of the backlash } \\
\text { parameter }(\propto)\end{array}$ & $\begin{array}{c}\text { Value of the distortion } \\
\text { factor }(D)\end{array}$ \\
\hline$\propto=0$ (healthy valve) & $D=1$ \\
\hline$\propto=2$ (fault valve) & $D=1.3$ \\
\hline$\propto=4$ (fault valve) & $D=1.72$ \\
\hline$\propto=6$ (fault valve) & $D=2.23$ \\
\hline
\end{tabular}

Table 1 The values of the distortion factor $D$ for different values of the backlash parameter $\propto$

\section{CONCLUSIONS}

In this paper we developed a new approach of the fault detection and diagnosis of the valve actuators for HVAC systems. This approach is very simple and more practical compared with other spectral approaches such as non-linear time series analysis, Fast Fourier Transform FFT, Short Fourier Transform STFT, or Wigner-Ville spectrum [1].

\section{REFERENCES}

[1] N.F.Thornhill , S.L. Shah, B.Huang , "Detection of Distribution Oscillations and Root-Cause Diagnosis", Proceedings of the IFAC Workshop on on-line Fault Detection and Supervision in the chemical process Industries, JeJudo Island, Korea, June 2001, pp. 167-172.

[2] N.Tudoroiu, M.Zaheeruddin, "SISO Neurocontrol Strategy Based on Inverse Neuro model for Improving Discharge Air Temperature (DAT) Performance", The $27^{\text {th }}$ Annual Congress of the American Romanian Academy of Arts and Sciences (ARA), Proceedings, Oradea, Romania, 2002.

[3] N.Tudoroiu, M.Zaheeruddin, R.V.Patel, "Linear and Nonlinear MIMO ARMA Models for Heating Ventilating and Air-Conditioning (HVAC)", The $26^{\text {th }}$ Annual Congress of the American Romanian Academy of Arts and Sciences (ARA), Proceedings, Montreal, Canada, 2001, pp. 525-530.

[4] M.Zaheeruddin.,R.V.Patel, “Optimal Tracking Control of Multi-Zone Indoor Environmental Space”, ASME Journal Dynamic Systems, Measurement and Control, Vol. 117, pp. 292-303, 1995.

[5] M.Zaheeruddin, G.R.Zheng, "A Dynamic Model of a Multizone VAV System for Control Analysis”, ASHRAE Transactions, Vol.100, Part 1, 1994.

[6] N.Tudoroiu, M.Zaheeruddin, "Frequency Analysis Approach to Detect and Diagnosis Faults in Discharge Air Temperature (DAT) Systems", the $30^{\text {th }}$ Annual ARA Congress, July 5-10, 2005, Chisinau, Republic of Moldova, pp.597-600.

[7] N.Tudoroiu, M.Zaheeruddin, “ Fault Detection and Diagnosis of Valve Actuators in HVAC Systems", Proceeding of the 2005 IEEE International Conference on Control Applications, August 29-31, 2005, Toronto, Canada, CD-ROM, IEEE Catalog \#:05CH37713C, ISBN:07803-9354-6

[8] N.Tudoroiu, K.Khorasani, "Fault Detection and Diagnosis for Satellite's Attitude Control System using an Interactive Multiple Model (IMM) Approach", Proceeding of the 2005 IEEE International Conference on Control Applications, August 29-31, 2005, Toronto, Canada, CD-ROM, IEEE Catalog \#:05CH37713C, ISBN:0-7803-93546. 